\title{
Microalgas asociadas a un macrófito flotante libre en un ambiente somero periurbano de la provincia de Corrientes (Argentina)
}

Nduré, N.L. ${ }^{1}$, Vallejos, S.V. ${ }^{1,2}$, Agustini, K. D. ${ }^{1}$, Gómez, F.D. ${ }^{1}$

\section{Resumen}

En este trabajo se presenta un estudio ecológico de microalgas asociadas a un macrófito flotante libre en la zona litoral de una laguna somera periurbana $\left(27^{\circ} 29^{\prime} \mathrm{S}\right.$; $58^{\circ} 45^{\prime} \mathrm{O}$ ) del $\mathrm{NO}$ de la provincia de $\mathrm{Co}^{-}$ rrientes. Se realizaron muestreos estacionales del macrófito-substrato y se registraron las principales variables ambientales: $\mathrm{pH}$, temperatura $\left({ }^{\circ} \mathrm{C}\right)$, conductividad $(\mu \mathrm{S} . \mathrm{cm}-$ $\left.{ }^{1}\right)$, oxígeno $(\mathrm{mg} / \mathrm{L})$, entre Marzo de 2015 y Febrero 2016. Para el estudio de la comunidad asociada a la vegetación se tomaron por triplicado muestras estacionales de la planta substrato (macrófito flotante libre); las que fueron conservadas con formaldehido al 4\% para su análisis cuantitativo y cualitativo en laboratorio. Las determinaciones taxonómicas se realizaron en base a la bibliografía general y específica. Se identificaron 39 taxones distribuidos en cinco grupos taxonómicos de microalgas fotosintéticas: Cyanobacteria (13), Glaucophyta (1), Chlorophyta (17), Bacillariophyta (4), Xantophyta (4). Chlorophyta fue el mejor grupo representado, aportando a la comunidad con un 57\% al total, seguida en orden de importancia las cianobacterias con el 30\%. Los otros grupos se encontraron ocasionalmente y en bajo número, aportando con el 13\%. La abundancia de la comunidad asociada estuvo relacionada con su posición en la planta substrato y las principales variables ambientales del cuerpo de agua. Este trabajo permite ampliar la información de microalgas asociadas a substratos naturales y representa una importante contribución al estudio de la ficoflora asociada a la vegetación acuática de la región del Nordeste Argentino.

Palabras Claves: Cyanobacteria, Perifitón, Bioindicador, variables ambientales 


\section{Abstract:}

This paper presents an ecological study of microalgae associated with a free floating macrophyte in the coastal zone of a shallow peri-urban lagoon $\left(27^{\circ} 29^{\prime} \mathrm{S}, 58^{\circ} 45^{\prime} \mathrm{W}\right)$ of the NW of the province of Corrientes. Seasonal sampling of the macrophyte-substrate was carried out and the main environmental variables were recorded: $p H$, temperature $\left({ }^{\circ} C\right)$, conductivity $\left(\mu S . \mathrm{cm}^{1}\right)$, oxygen ( $m g / L)$, between March 2015 and February 2016. For the study of the community associated with the vegetation were taken in triplicate seasonal samples of the substrate plant (free floating macrophyte); those that were preserved with 4\% formaldehyde for their quantitative and qualitative analysis in the laboratory. The taxonomic determinations were made $b a-$ sed on the general and specific bibliography. We identified 39 taxa distributed in five taxonomic groups of photosynthetic microalgae: Cyanobacteria (13), Glaucophyta (1), Chlorophyta (17), Bacillariophyta (4), Xantophyta (4). Chlorophyta was the best represented group, contributing to the community with 57\% of the total, followed in order of importance followed by cyanobacteria with 30\%. The other groups were found occasionally and in low numbers, contributing with 13\%. The abundance of the associated community was related to its position in the substrate plant and the main environmental variables of the water body. This work makes it possible to expand the information on microalgae associated with natural substrates and represents an important contribution to the study of the phytophalous associated with the aquatic vegetation of the Northeast Argentine region.

Keywords: Cyanobacteria, Perifitón, Bioindicator, environmental variables

\section{Introducción:}

En ambientes tropicales y subtropicales los estudios taxonómicos de microalgas asociadas o adheridas a la vegetación son escasos y fragmentarios, particularmente en substratos naturales (Tell 1973, Alvarez et al., 2000; Pizarro, 1991, 2008; Wenzel y Díaz; 2008; Vallejos, 2008; Martínez ,2010; Vallejos,2014). Las lagunas pertenecientes a la Cuenca del Riachuelo (Corrientes, Argentina) se caracterizan por presentar diversos grupos ecológicos de macrófitos que sustentan una variada microflora fotosintética de cianobacterias y algas (Vallejos, 2014). La Cuenca del Riachuelo forma parte de una de las seis grandes regiones de humedales de Argentina (Cuenca del Plata), con gran influencia antrópica e importantes áreas de desarrollo agrícola ganadero (Canevari et al., 1998). Entre las comunidades más importantes adheridas o asociadas a la vegetación acuática se destaca el perifiton (Wetzel, 1983). El perifiton es de gran importancia ecológica como productor de materia orgánica y por su rol en el metabolismo de los ecosistemas acuáticos (Pompêo y Moschini-Carlos, 2003). Esta comunidad debido a su estabilidad es utilizada frecuentemente como bioindicadora de la calidad del agua (Pizarro, 2005). Algunos autores denominan biopelícula o biofilm al conjunto de organismos autótrofos y he- 
terótros que interactúan entre sí dentro de una matriz mucilaginosa, representando las algas, un componente importante de esta biopelícula. Hasta el momento la mayoría de las cianobacterias registradas para el NE Argentino fueron incluidas en estudios del fitoplancton (Tell 1994; Zalocar de Domitrovic y Forastier,2005; Forastier, 2012) y recientemente en estudios del perifiton (Vallejos, 2008; Martínez, 2010; Vallejos, 2014; Vallejos et al., 2015).

Entre los organismos fotosintéticos que integran la comunidad de microalgas asociadas a la vegetación, el estudio de las cianobacterias y su representatividad en el sistema (abundancia y variaciones temporales) merece especial atención por presentar especies señaladas a nivel mundial como potencialmente toxígenas (Chorus y Bartram 1999) y por haberse registrado recientemente su presencia en ambientes del Nordeste Argentino (Vallejos,2014).

En este trabajo se presenta un estudio ecológico de las microalgas fotosintéticas asociado a un macrófito flotante libre, analizando sus variaciones estacionales de abundancia en relación a la planta substrato $\mathrm{y}$ a las principales variables ambientales.

\section{Materiales y Métodos:}

Para el estudio de las microalgas fotosintéticas se seleccionó una laguna periurbana en cercanías de Corrientes capital, donde se colectaron muestras estacionales entre Marzo 2015 y Febrero de 2016.

Se seleccionó un macrófito flotante libre dominante en la zona litoral de la lagu- na. Para el análisis cualitativo se tomaron por triplicado muestras estacionales de la planta substrato. En el laboratorio, para el análisis cualitativo se observó el material vivo al microscopio de luz por observación directa. Posteriormente se fijó y conservó en FAA o formaldehído al 4\%. La determinación taxonómica de los grupos de microalgas que se encuentran adheridos o asociados al macrófito substrato se realizó en base a obras clásicas de Bourrelly (1968, 1970, 1972) y Bicudo y Menezes (2006). Las determinaciones genéricas e infra específicas de cianobacterias se realizaron en base a la clasificación de Anagnostidis y Komárek (1990) y Komárek y Anagnostidis $(1989,1998,2005)$ hasta categoría de especie en los casos que fueron posible.

Para el análisis cuantitativo de microalgas se siguió la metodología utilizada por Pozzobon y Tell, 1995. En cada muestreo se colectaron 30 plantas de la zona litoral del cuerpo de agua (Fig.2). De cada muestra estacional se tomaron 3 plantas al azar y de cada planta se extrajeron 3 escamas del centro y 3 de la periferia bajo microscopio estereoscópico. De cada escama se analizaron 6 campos (400x), 2 de la base de la escama inferior (EI), 2 del centro (EC) y 2 del extremo superior (ES). Se observaron en total 54 campos de las escamas del centro, un total de 36 campos de las escamas superiores y 18 campos en total de la escama inferior.

El número de algas y cianobacterias promedio será referido a la superficie substrato como $\mathrm{N}^{\circ}$ de ind. $\mathrm{cm}-2$ (Schwarzbold, 1990). Las cianobacterias y microalgas 
fotosintéticas fueron fotografiadas en $\mathrm{mi}-$ croscopio Leica de luz convencional con cámara incorporada.

Durante las operaciones de campaña, paralelamente a la extracción de las muestras, se tomaron mediciones in situ de las principales variables ambientales tenidas en cuenta para el desarrollo de la comunidad: transparencia (estimada con disco de Secchi de $20 \mathrm{~cm}$ de diámetro), temperatura y $\mathrm{pH}$ (con peachímetro digital Metrohm A G Herisau). La diversidad específica fue calculada mediante la aplicación del índice de Shannon y Weaver Weaver (Shannon y Weaver, 1963). Se empleo el software PAST (Hammer, 2001) para el procesamiento de la información.

\section{Resultados}

A continuación, se describen los resultados obtenidos de las variables ambientales registrados en la zona litoral de la laguna durante los períodos de muestreo.

Temperatura: En general, la temperatura del agua siguió un patrón estacional; registrándose una mínima de $21{ }^{\circ} \mathrm{C}$ en otoño (25/05/2015), a $50 \mathrm{~cm}$ de profundidad y una máxima en verano de $33^{\circ} \mathrm{C}$ (29/02/2016), a partir de los $70 \mathrm{~cm}$ de profundidad.

Oxígeno: Las concentraciones de oxígeno disueltos arrojaron valores entre 6-8 $\mathrm{mg} / \mathrm{L}$ en los distintos períodos de estudios, encontrándose dentro de los rangos aceptables para la vida acuática.
Conductividad: La conductividad eléctrica para la estación de otoño presentó un valor mínimo de $45 \mu \mathrm{S} . \mathrm{cm}-1$ y un valor máximo de $68 \mu \mathrm{S} . \mathrm{cm}-1$ para la estación de verano.

pH: durante los muestreos estacionales el valor promedio que arrojo 7,95 (alcalino). El valor máximo se registró en el muestreo de otoño con un $\mathrm{pH}:(8,8)$ en 25/05/2015.

\section{Composición y variaciones estacio- nales de las microalgas}

Se registró la presencia de cinco grupos taxonómicos de microalgas fotosínteticas: Cyanobacteria, Glaucophyta, Chlorophyta, Bacillariophyta y Xanthophyta.

Chlorophyta fue el grupo mejor representado en riqueza de especies (17) y densidad entre 29 y 594 ind.cm-2, seguido en importancia el grupo de las Cyanobacteria (13) y densidad entre $56(29 / 02 / 16)$ y 294 ind.cm-2 durante el verano (29/2/16) y primavera (29/11/15) respectivamente. Los grupos de Glaucophyta, Bacillariophyta y Xanthophyta solo se encontraron ocasionalmente y en bajo número. La diversidad específica de las microalgas asociadas al macrófito flotante libre varió entre 1,7 y 2,2 bits.ind-1. En la tabla 1 se presenta un listado de grupos taxonómicos más representativos asociados al substrato, señalando el número de taxones de cada grupo, especies y hábitat. 


\begin{tabular}{|c|c|c|c|c|}
\hline \multirow{2}{*}{ División } & \multirow[b]{2}{*}{ Especie } & \multicolumn{3}{|c|}{ Hábitat } \\
\hline & & $\mathrm{Pl}$ & $\mathrm{Pe}$ & $\mathrm{Be}$ \\
\hline $\begin{array}{c}\text { Cyanophyta } \\
\text { (13) }\end{array}$ & $\begin{array}{l}\text { Anabaena sp. } \\
\text { Aphanothece stagnina (Sprengel) A. Braun. } \\
\text { Calothrix sp. } 1 \\
\text { Calothrix sp. } 2 \\
\text { Calothrix sp. } 3 \\
\text { Leptolyngbya Anagnostidis y Komárek. } \\
\text { Leptolyngya perelegans (Lemmermann) Anagnostidis et } \\
\text { Komárek } \\
\text { Leptolyngbya chlorina (Perfilev) Anagnostidis et Komárek. } \\
\text { Microchaete uberrima. Carter. } \\
\text { Nostoc sp. } \\
\text { Nostoc punctiforme (Kützing ex Hariot) Hariot. } \\
\text { Spirulina sp. } \\
\text { Stigonema hormoides (Kütz.) Bornet y Flahault. }\end{array}$ & $\begin{array}{l}\mathrm{X} \\
\mathrm{x} \\
\mathrm{x} \\
\\
\mathrm{x} \\
\mathrm{x}\end{array}$ & $\begin{array}{l}x \\
x \\
x \\
x \\
x \\
x \\
x \\
x \\
x \\
x \\
x \\
x \\
x\end{array}$ & $\begin{array}{l}X \\
x\end{array}$ \\
\hline $\begin{array}{c}\text { Glaucophyta } \\
\text { (1) }\end{array}$ & Glaucocystis nostochinearum (Itzigsohn)Rabenh. & $\mathrm{X}$ & & \\
\hline $\begin{array}{c}\text { Chlorophyta } \\
\text { (17) }\end{array}$ & $\begin{array}{l}\text { Bulbochaete sp. } \\
\text { Coleochaete scutata Brébisson. } \\
\text { Coleochaete soluta. Brébisson. } \\
\text { Coleochaete pulvinata.A.Braun.. } \\
\text { Cosmarium sp. } \\
\text { Euastrum turgidum Ehrenberg ex Ralfs. } \\
\text { Eudorina sp. } \\
\text { Kirchneriella lunaris Schmidle. } \\
\text { Mougeotia sp. } \\
\text { Oedog̣onium sp. }\end{array}$ & $\begin{array}{l}x \\
x\end{array}$ & $\begin{array}{l}x \\
x \\
x \\
x \\
x\end{array}$ & \\
\hline
\end{tabular}




\begin{tabular}{|c|c|c|c|}
\hline & $\begin{array}{l}\text { Oedog̣onium sp. } \\
\text { Oedog̣onium capillare Kützing ex Hirn } \\
\text { Oedog̣onium reinschii Rov.J.de Hirn } \\
\text { Oocystis lacustris Nägeli. } \\
\text { Stigeoclonium tennue Kutzing. } \\
\text { Spirogyra sp. } \\
\text { Zyg̣nema sp. } \\
\text { Apatococcus lobatus (Chodat) J. B. Petersen. }\end{array}$ & $\begin{array}{l}X \\
x\end{array}$ & $x$ \\
\hline $\begin{array}{c}\text { Xanthophyta } \\
\text { (4) }\end{array}$ & $\begin{array}{l}\text { Centritractus Lemmermann } \\
\text { Characiopsis Borzi } \\
\text { Ophiocytium capitatum Wolle } \\
\text { Ophiocytium maius Nägeli }\end{array}$ & $\begin{array}{l}X \\
X\end{array}$ & $x$ \\
\hline $\begin{array}{c}\text { Bacillariophyta } \\
\text { (4) }\end{array}$ & $\begin{array}{l}\text { Eunotia Ehrenberg. } \\
\text { Gomphonema Ehrenberg. } \\
\text { Navicula Borv. } \\
\text { Pinnularia Ehrenberg. }\end{array}$ & $\begin{array}{l}X \\
X\end{array}$ & $x$ \\
\hline
\end{tabular}

El grupo dominante, representado por Chlorophyta, estuvo integrado con mayor abundancia por el orden Oedogoniales con dominancia de Oedogonium spp., y especies estrictamente epífitas de Chaetophorales como Coleochete scutata, Coleochaete soluta y Coleochaete pulvinata. Cyanobacteria fue el grupo subdominante con formas, filamentosas de Oscillatoriales como Leptolyngbya spp., entre ellas Leptolyngbya chlorina, especie registrada por primera vez en ambientes de la cuenca del Riachuelo y Nostocales como Microchaete uberrina.

Se observaron variaciones estacionales en la composición de la comunidad de mi- croalgas asociadas a un macrófito flotante libre, de acuerdo a la posición en la planta substrato y las principales variables ambientales

\section{Discusión:}

Trabajos previos realizados en el fitoplancton en dicho ambiente acuático registraron la presencia de cianobacterias y clorofíceas (Forastier, 2012) o mencionan algunas especies del perifiton en el fitoplancton de este cuerpo de agua (Zalocar et al., 1998). Otros autores registraron microalgas fotosintéticas de los grupos: Cyanobacteria y Chlorophyta epifitas, como 
dominantes en ambientes temporarios de la cuenca del Riachuelo, provincia de Corrientes (Tell y Pizarro, 1984) o asociadas a Azolla caroliniana (Pizarro, 1991). Estudios realizados en el perifiton de Potamogeton sp. (Vallejos, 2008) y Nymphoides indica (Martínez, 2010) en la laguna Aeroclub (Cuenca del Riachuelo, Corrientes) señalaron seis grupos taxonómicos y sus variaciones espaciales y temporales en el perifiton teniendo en cuenta las principales variables ambientales. Las características limnológicas registradas en este estudio fueron diferentes a las señalas por Zalocar et al., 1998, en el fitoplancton del mismo cuerpo de agua en el que determinaron ocho grandes grupos taxonómicos de microalgas; de las cuales solo un grupo fue registrado nuevamente en este trabajo: Chlorophyceae.

La diversidad y abundancia de los taxones de microalgas perifíticas pueden estar asociadas a diversos factores ambientales $\left(\mathrm{T}^{\circ}, \mathrm{pH}\right.$, nutrientes, oxígeno, conductividad, luz, macrófitos, morfometría del cuerpo de agua), aspectos que fueron señalados por Zalocar et al., 2013.

Las características limnológicas registradas en la presente investigación fueron similares a las descriptas anteriormente por Forastier, (2012) que señala que las variaciones de abundancia en ambos grupos taxonómicos están en relación a la elevada transparencia del agua en este cuerpo. Asimismo Vallejos (2014) señala a la temperatura del agua, como condicionante de las concentraciones de oxígeno y dióxido de carbono lo que concuerda con los resultados obtenidos en este análisis en el cual los valores de concentración de oxígeno están dentro de los rangos aceptables para la vida (6-8mg/1). Zalocar et al., (1998), en cambio describe un $\mathrm{pH}$ ácido para la laguna, no coincidente con este trabajo, ya que el $\mathrm{pH}$ obtenido presentó valores dentro del rango alcalino $(8,8)$.

\section{Conclusiones}

Asociados al macrófito substrato analizado ,se registraron durante el período de estudios 29 géneros y 38 especies de algas perifíticas agrupados en cinco grupos taxonómicos: Chlorophyta (17), Cyanobacteria (13), Bacillariophyta (4), Xantophyta (4) y Glaucophyta(1).

De las doce submuestras analizadas, Chlorophyta resultó ser el grupo más abundante, aportando a la comunidad con un $57,3 \%$. Dentro de ese grupo las formas filamentosas estuvieron mejor representadas por el género Oedogonium, que presento mayor abundancia y diversidad, en los meses de primavera (594 ind.cm-2) preferentemente donde se observó la colonización de la parte central de cada escama hacia la periferia de las mismas. Se registraron tres especies del género Coleochaete de las cuales dos son citadas por primera vez para la provincia de Corrientes: Coleochaete soluta y Coleochaete pulvinata. Así mismo sucedió con el género Oedogonium para el cual se registró dos nuevas especies para la provincia (Oedogonium.capillare y O.reinschii) y también Apatococcus lobatus señaladas por primera vez; esto demuestra la gran diversidad de clorófitos.

En total se identificaron 13 taxones de 
cianobacterias distribuidos en 8 géneros pertenecientes a los ordenes: Synechococcales, Spirulinales Oscillatoriales y Nostocales, este grupo representó más del 30\% del total de algas perifíticas y el $38 \%$ correspondió al orden Nostocales; siguieron el mismo patrón de colonización que Chlorophyta, encontrándose también en mayor abundancia en los meses de primavera (294 ind.cm-2). El grupo Glaucophyta aportó con un $0,2 \%$ del total a la comunidad de las microalgas perifiticas, con presencia en los muestreos de invierno, primavera y verano. Bacillariophyta contribuyó con el $11 \%$ al grupo total; la mayoría de los individuos fueron identificadas a nivel de género debido a su baja frecuencia en las submuestras analizadas (Navicula y Pinnularia, Eunotia y Gomphonema). En el caso de Xantophyta solo contribuyo con $0,6 \%$ del total, encontrándose ausente en los meses de verano.

Los resultados de este trabajo amplían la información acerca de la composición y distribución de microalgas fotosintéticas asociadas a substratos naturales en función de la posición en la planta substrato (escamas) y aporta datos acerca de las variaciones temporales en función a las principales variables ambientales entre los años 2015 y 2016.

\section{Bibliografía:}

Álvarez, S.V., G.I. Bazan y M.T. Wenzel. 2000. Hormogonales (Cyanophyta) de la Laguna el Guanaco (La Pampa, Argentina). Darwiniana, 38:279-284p.

Anagnostidis, K. y J. Komárek. 1990. Modern approach to the classification system of cyanophytes.5-Stigonematales.Archiv Hidrobiol./Suppl.86,Algological Studies 59:1$73 \mathrm{p}$.

Bicudo, D.C. y M. Menezes. 2006. Gêneros de algas de águas continentais do Brasil:chave para identificacão e descricões.RIMA,São Carlos.

Bourrelly, P. 1968. Les alguesd'eaudouce. II: Les alguesjaunes et brunes. N. Boubeé.,París. $438 \mathrm{p}$.

Bourrelly, P. 1970. Les alguesd'eaudouce. III: Les alguesbleues et rouges. N. Boubeé,París, 512 p.
Bourrelly, P. 1972. Les alguesd'eaudouce. I: Les alguesvertes. N. Boubeé, París, 572p.

Carnevali ,R. 1994. Fitogeografía de la Provincia de Corrientes. Gobierno de la

Provincia de Corrientes. Instituto Nacional de Tecnología Agropecuaria, Corrientes, 324p.

Chorus, I. y J. Bartram (eds.).1999. Toxic Cyanobacteria in wáter.A guide to their public health consequences, monitoring and management.WHO.E y FN Spon,London,416p. Forastier ,M.E. 2012. Diversidad y toxicidad de Cyanophyta (Cyanobacteria) del Nordeste Argentino. Tesis Doctoral, Universidad Nacional del Nordeste, Corrientes, 207 p.

García de Emiliani,M.O; 1980.Fitoplancton de una laguna del valle aluvial del Paraná Medio(“Los Matadores”,SantaFé,Argentin a).I.Estructura y distribución en relación a 
factores ambientales. Ecología 4.127-140p. Hammer, O., D.A.T. Harper y P.D. Ryan. 2001. Past: Paleontological Statistics Software Package for Education and Data Analysis. Paleontología Electronica, 4:1-9p.

Komárek, J. y K. Anagnostidis.1989.Modern approach to the classification system of cyanophytes.4-Nostocales.Archiv fürHidrobiologie/Supplementband 82, Algological Studies 56:247-345p

Martínez, M.S. 2010. Microalgas fotosintéticas asociadas a Nymphoides indica (L.) Kuntze en una laguna del NO de la provincia de Corrientes (Argentina). Tesis de Grado, Universidad Nacional del Nordeste. 27 p.

Pizarro, H. N. 1991. Algas nuevas o interesantes asociadas a la vegetación acuática de ambientes Leníticos de la provincia de Corrientes (Argentina). Bol.Soc.Arg.Botanica, 27(1-2):31-35p.

Pompěo, M. L. M. y C. Moschini- V. 2003. Macrófitas aquaticas e perifíton, aspectos ecológicos e metodológico. RIMA, Săo Carlos, 134p.

Schwarzbold, A. 1990.Métodos ecológicos aplicados ao estudo do perifiton. Acta Limnol.Brasil.3:545-592.

Shannon, C.E. y W. Weaver.1963. The mathematical theory of communication. Illinois University Press, Urbana,177p.

Tell, G. 1973. Cyanophyta epífitas de las La- gunas Chascomús, El Burro, Yalca y Vitel (Prov. Buenos Aires, Argentina). Darwiniana, 18: 129-152p.

Tell,G. 1994. Planktonic Cyanophyceae from northeastern Argentina. Rev. Hydrobiol.

Trop., 27 (4): 347-360p.Anexo.

Vallejos, S.V. 2008.Cyanobacterias del perifiton de Potamogeton sp. en una Laguna de la Cuenca del Riachuelo (Corrientes, Argentina).Tesis de Grado, Universidad $\mathrm{Na}$ cional del Nordeste. 37 p.

Vallejos, S.V. 2014. Diversidad de Cianobacterias Asociadas a Micrófitos Acuáticos en Ambientes Someros del Nordeste Argentino. Tesis Doctoral, Universidad Nacional del Nordeste, Corrientes, $223 \mathrm{p}$.

Vallejos, S.V., Y.Zalocar de Domitrovic y M.S. Martínez.Periphytic algae of two bioforms of macrophytes in a subtropical shallow lake of Argentina.IHERINGIA, Ser.Bot; Porto Alegre, v.70, n.1, p.129-142, Junho 2015.

Wenzel, M.T. y M. M. Díaz.2008. Cyanophyta del Parque Nacional Nahuel Huapi(Argentina), II. Darwiniana, 46 (1):51-65p.

Zalocar de Domitrovic ,Y. y M.E .Forastier.2005.Biodiversidad de Cyanophyceae (Cyanobacteria) y especies toxigénicas del litoral fluvial argentino.INSUGEO, Miscelánea 14:213-228p. 